\title{
Cadaveric Study on Retrograde Calcaneotibial Fixation of Unstable Ankle Fractures
}

\author{
ELENA MARIE LOSCHAN ${ }^{1,2}$, PADHRAIG O'LOUGHLIN $^{3}$, CHRISTIAN KRETTEK $^{2}$ and RALPH GAULKE ${ }^{1,2}$ \\ ${ }^{1}$ Section Upper Extremity, Foot- and Rheuma Surgery, Trauma Department, \\ Medical School Hanover (MHH), Hannover, Germany; \\ ${ }^{2}$ Trauma Department, Medical School Hanover (MHH), Hannover, Germany; \\ ${ }^{3}$ Mater Hospital, Citygate, Mahon, Cork, Ireland
}

\begin{abstract}
Background/Aim: In extensive soft tissue injury, primary internal fracture fixation is not always possible and external fixation for primary immobilization plays an important role. The aim of this study was to compare the accuracy of freehand technique and of a target device for retrograde calcaneotibial fixation in an uninjured bone and an unstable ankle fracture. Materials and Methods: The calcaneotibial fixation was performed randomly by an experienced surgeon utilizing either a freehand drilling technique or a novel targeting device in four human cadavers before and after producing a bimalleolar ankle fracture. The duration of foot positioning, drilling, number of intensifier shots, and drilling attempts were recorded. The final position of the wire was established using IsoC-3D scan. Results: The mean for definitive wire placement with the target device was $247 \mathrm{sec}$ with a mean of 3.4 attempts, and $92 \mathrm{sec}$ and 2.1 attempts in the freehand technique. Mean intraosseous distance of the K-wire was $45.3 \mathrm{~mm}$ and 63.6 $\mathrm{mm}$ in the tibia and $39.7 \mathrm{~mm}$ and $41.5 \mathrm{~mm}$ in the calcaneus respectively. Conclusion: The novel target device for calcaneotibial fixation achieves similar results to a trained surgeon using the freehand technique.
\end{abstract}

In the setting of extensive soft tissue injury, primary fracture fixation of ankle fractures, following high energy trauma, is often not possible (1-4). However, immobilization in a lower

This article is freely accessible online.

Correspondence to: Prof. Dr. med. Ralph Gaulke, Leiter der Sektion Obere Extremität, Fuß- und Rheumachirurgie, Unfallchirurgische Klinik, Medizinische Hochschule Hannover, Carl-Neuberg-Str. 1, D-30625 Hannover, Germany. Tel: +49 5115322015, e-mail: gaulke.ralph@mh-hannover.de

Key Words: Calcaneotibial transfixation, ankle fractures, biomechanics, targeting device. leg cast carries the risk of pressure necrosis and loss of reduction of unstable fractures (5-7). Thus, tibiometatarsal external fixation has a well-established role in high-energy lower leg trauma care $(1,2,8-11)$. This technique facilitates secure stabilization whilst minimizing secondary iatrogenic injury to traumatized soft tissues $(12,13)$. However, there is a risk of pin track infection $(12,14-16)$ and a loss of reposition in instable fracture situation with external fixation $(8,14)$.

Retrograde trans-articular calcaneotalotibial K-wire fixation is an alternative mode of hindfoot fixation (17). It can also be used as an additional tool in insufficient stabilization of an ankle fracture by an external fixator. However, it does carry an inherent risk of cartilage damage of the subtalar and ankle joint $(12,17)$. Extra-articular calcaneotibial K-wire-fixation represents a worthy alternative, but it is technically demanding $(3,17)$. In a preliminary study, the current authors measured the ideal wire position, dimension of bony corridor as well as the optimal entry point and angle of the wire using standard radiographs (Figure 1) (18). The bony corridor keeps the risk of iatrogenic injuries very low. It does not run parallel to the neurovascular bundle and it does not cross it. Based on these values, a targeting device was developed, which enables safe wire placement based on established parameters (Figure 2). The aim of this study was to compare the utility of this device to a freehand technique.

\section{Materials and Methods}

The tests were performed using four alcohol-formalin-fixed cadavers (eight feet). Clinical examination revealed unrestricted mobility of the ankle and hindfoot joints and ligamentous stability. Fluoroscopy demonstrated no significant foot or ankle pathology. In the first series, a randomized trial was conducted on an uninjured foot and ankle. The free hand technique was performed on one side and the target device on the contralateral side. All K-wire fixations were performed by an experienced trauma and foot and ankle surgeon, who was firm in the freehand technique, but never used the target device before. The aim was to analyze if it is possible to 
a
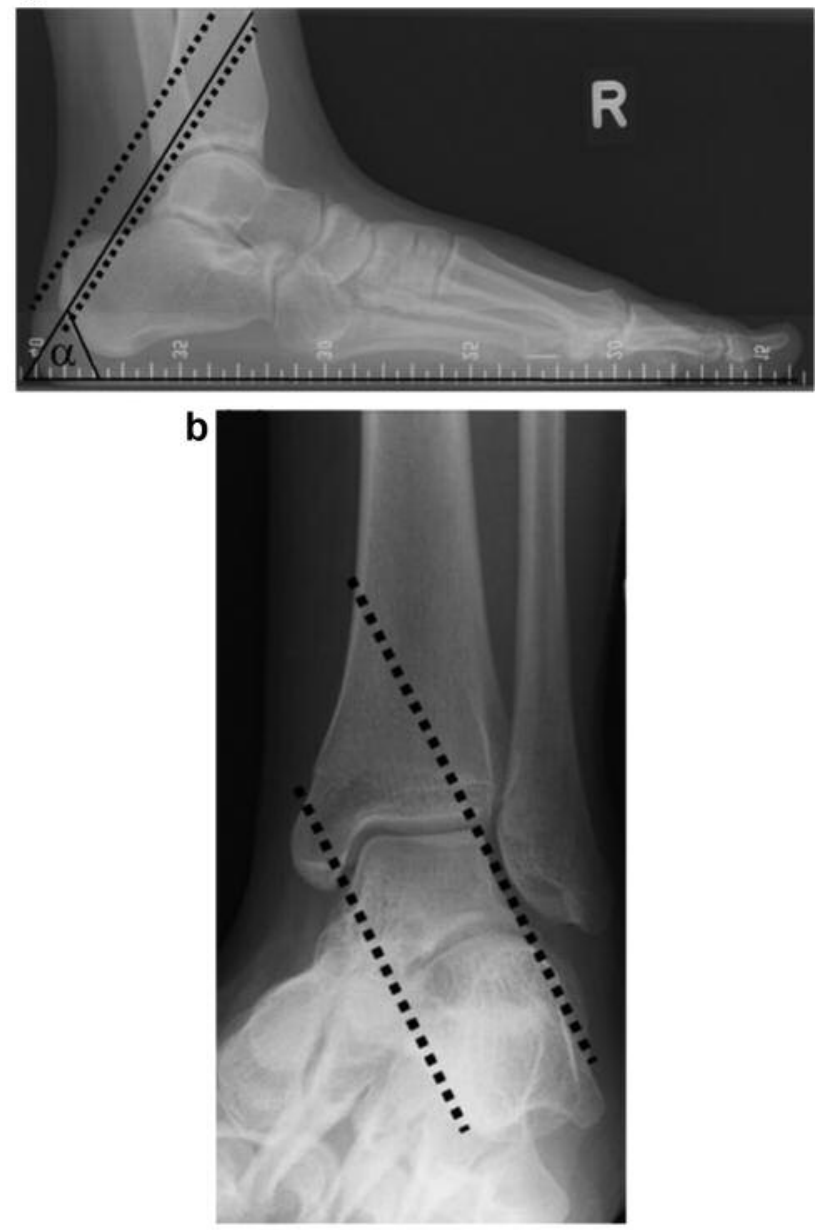

Figure 1. Corridor for proper K-wire placement in the sagittal (a) and coronar plane (b) (5).

receive match able results with the targeting device compared to the freehand technique performed by an experienced surgeon. The foot positioning time (including image intensifier set-up for a lateral projection) for the target device group, was recorded. In both groups, the time taken for correct wire insertion with image intensifier (in two planes) was recorded. Furthermore, the number of drilling attempts and the number of image intensifier shots before, during and after drilling were collected. Afterwards, a 3D CT scan of the hindfoot was performed using three-dimensional imaging with a motorized mobile C-Arm (Siremobil Iso-C 3D, Siemens AG, Munich, Germany, Figure 3). In the second test series, the experiments were repeated following the same protocol, but on a specimen with an unstable bimalleolar fracture of the ankle joint, which had been produced using an osteotome (Figure 3).

Subsequently, the image intensifier images and the Iso-C 3D images were analyzed using OsiriX imaging software (OsiriX, version 3.8.1) in the sagittal plane, coronal plane and in the plane of the wire. The following measurements in the sagittal plane (lateral view) were recorded: the length of the wire in the calcaneus in the plane of the wire as well as measurement of the maximum distance to the posterior calcaneal border rectangular to the wire, the length of the wire in the tibia and the distance between the border of the distal tibia joint surface and the tibial entry point and whether the wire was located within the corridor defined by Schröder et al. (18) (Figure 4). In the coronal plane (anteroposterior view), the following parameters were assessed: the width of the distal tibial articulation, the distance between the lateral tibial border and the wire entry point. From these values a ratio was calculated to enable a comparison of ankles of different sizes. Furthermore, the distance between the nail exit from the tibia and the distal tibial surface was determined.

\section{Results}

In the first series (four pairs of intact specimens), using the targeting device, the mean $\mathrm{K}$-wire positioning time in the intact bone was 162 (range=140-179) sec. The mean number of drilling attempts was 4.5 (range $=1-9$ ) and took a mean of 378 (range $=80-768$ ) sec to perform. In the freehand drilling group, the mean number of drilling attempts were 2.3 (range $=1-4$ ) and took a mean of $140 \mathrm{sec}($ range $=32-365 \mathrm{sec}) \mathrm{sec}$ to perform. The mean required number of image intensifier shots was 27 (range=5-49) with the target device and 15 (range=4-19) with the freehand drilling technique respectively (Table I).

In the second series, with four pairs of unstable bimalleolar fractures, foot positioning into the targeting device took a mean of $144 \mathrm{sec}$ (range $=122-160 \mathrm{sec}$ ). A mean of 2.3 (range=1-4) drilling attempts were needed for correct wire placement with a mean of $116 \mathrm{sec}$ (range $=36-194 \mathrm{sec})$. Using the freehand technique, it took a mean of $43 \mathrm{sec}$ (range $=23-71 \mathrm{sec}$ ) and a mean of 2 (range=1-4) drilling attempts to place the K-wire in the correct position. The mean required number of image intensifier shots was 8 (range=4-12) with target device and 4.8 (range=3-8) with the freehand drilling technique respectively (Table I).

Overall, in both series, the mean time for positioning of the foot within the device was $153 \mathrm{sec}$ (range $=122-179 \mathrm{sec}$ ) and 247 $\mathrm{sec}$ (range $=36-768 \mathrm{sec}$ ) for proper wire placement, respectively. The mean number of drilling attempts was 3.4 (range=1-9), whereas the mean number of image intensifier shots was 17.4 (range=4-49). With the free hand technique, the mean time for proper K-wire placement was $92 \mathrm{sec}$ (range=23-365 sec) for a mean of 2.1 (range $=1-4)$ required attempts. The mean number of image intensifier shots required was 9.9 (range $=3-25)$. The time for positioning and drilling as well as the number of drilling attempts decreased within the series (Figure 5).

All freehand-placed wires were correctly positioned within the corridor described by Schröder et al. (18). Using the device, the mean intra-tibial and intra-calcaneal distances of the wires were $45.3 \mathrm{~mm}$ (range $=26.2$ to $58.2 \mathrm{~mm}$ ) and 39.7 $\mathrm{mm}$ (range=30.0-58.9 mm), respectively. After freehand drilling, the mean intra-tibial and intra-calcaneal distances were $63.6 \mathrm{~mm}$ (range $=44.1-83.0 \mathrm{~mm}$ ) and $41.4 \mathrm{~mm}$ 
a

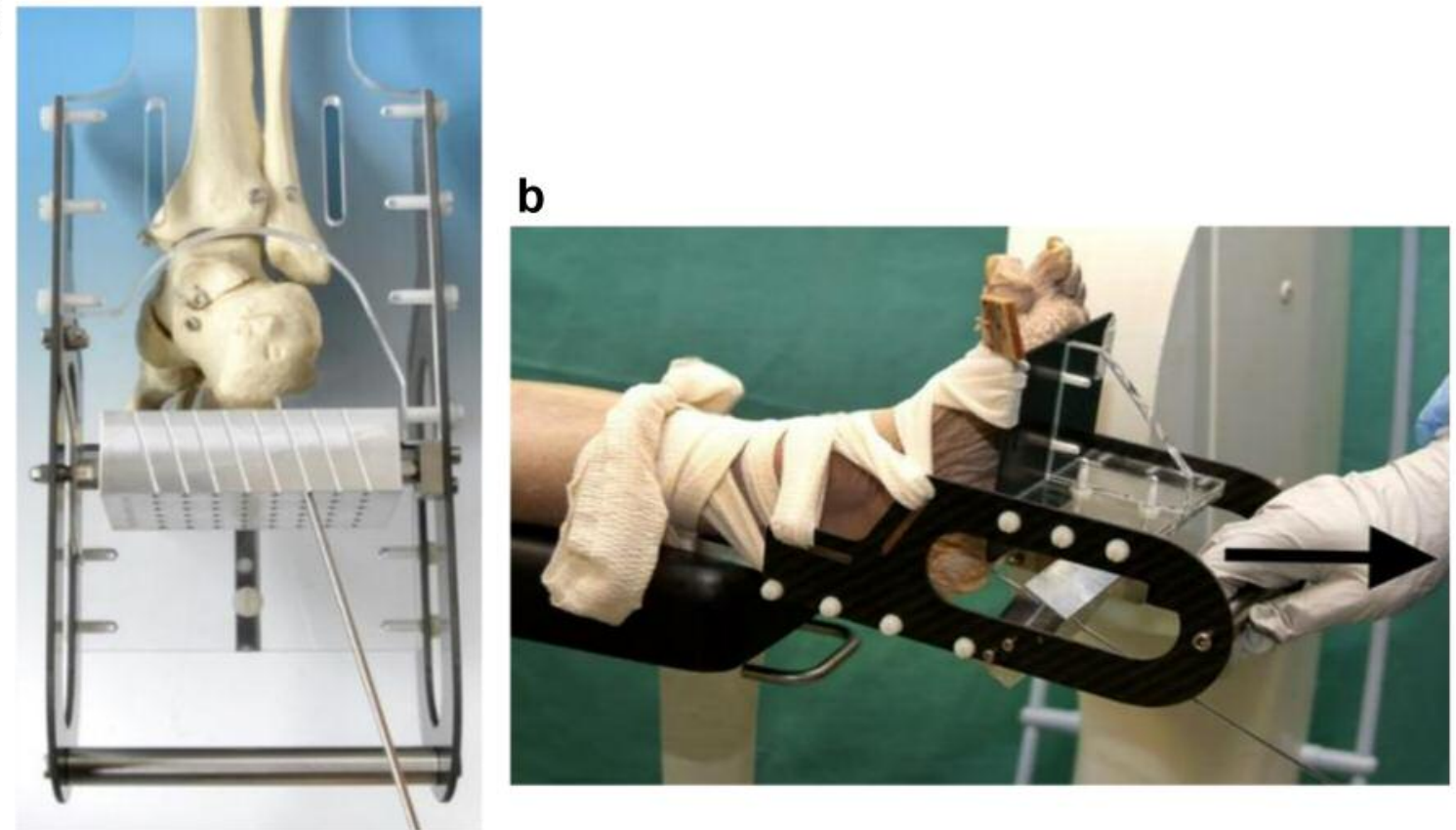

Figure 2. Target device with an artificial bone (a) and with a cadaver specimen (b). Reposition under manual traction (arrow).

a

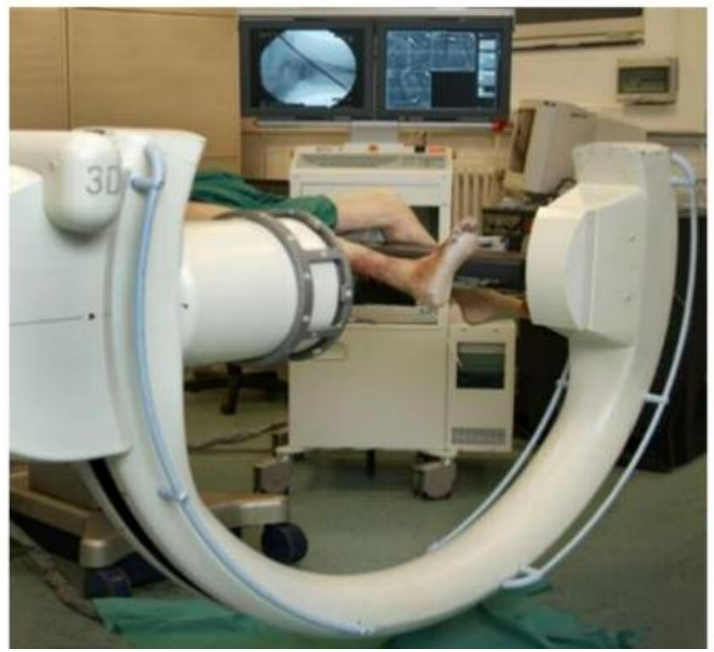

b

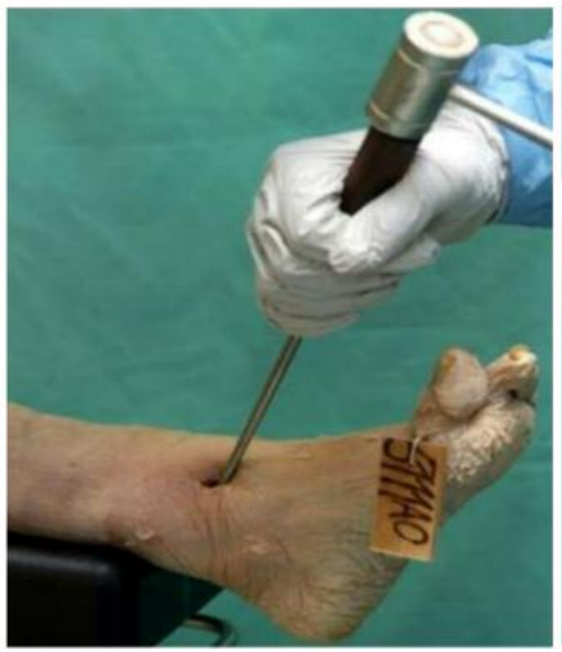

C

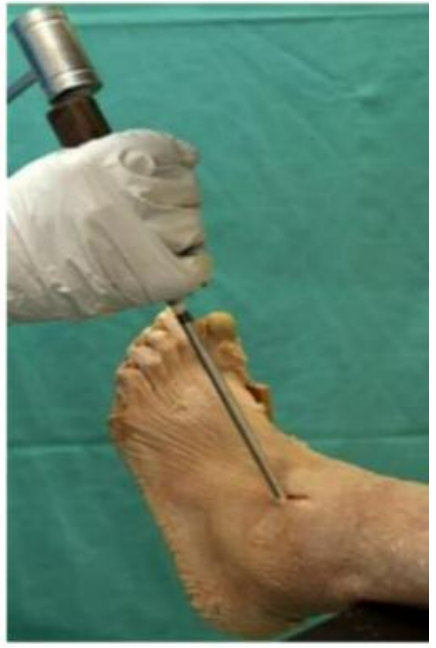

Figure 3. Setup with a cadaver specimen (a), percutaneous osteotomy of the medial (b) and lateral malleolus (c).

(range=33.0-47.6 mm), respectively. In one case of both groups, the proximal tibial outlet point could not be successfully defined in the x-rays. The average tibial track was significantly shorter when drilling with the aid of the device than with the freehand technique $(p=0.015)$. The investigators did not find any significant difference between the groups when assessing the calcaneal track $(p=0.303)$.
Correct positioning of the wire within the corridor was found in all cases with the freehand technique.

Using the targeting device, all wires were placed correctly in the lateral corridor. In one fracture case the coronal corridor was missed, although the wire had an intraosseous position (Table II). In this case the wrong drill sleeve was chosen, resulting in an entry point located too lateral (Figures 2 and 6). 


\section{a}

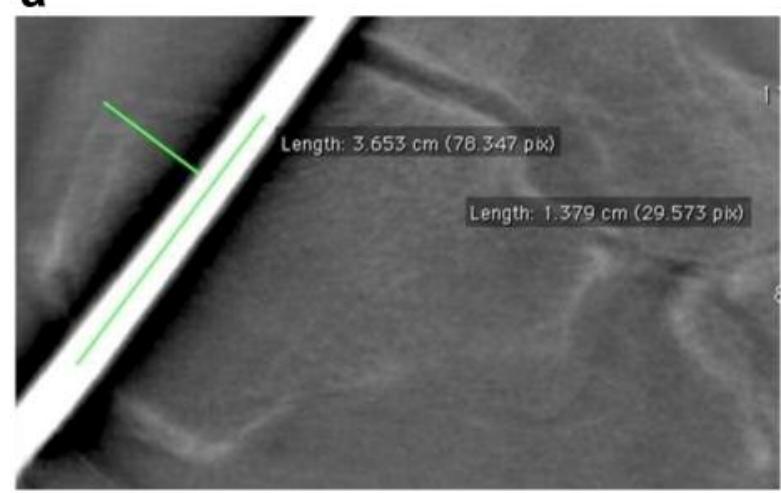

b

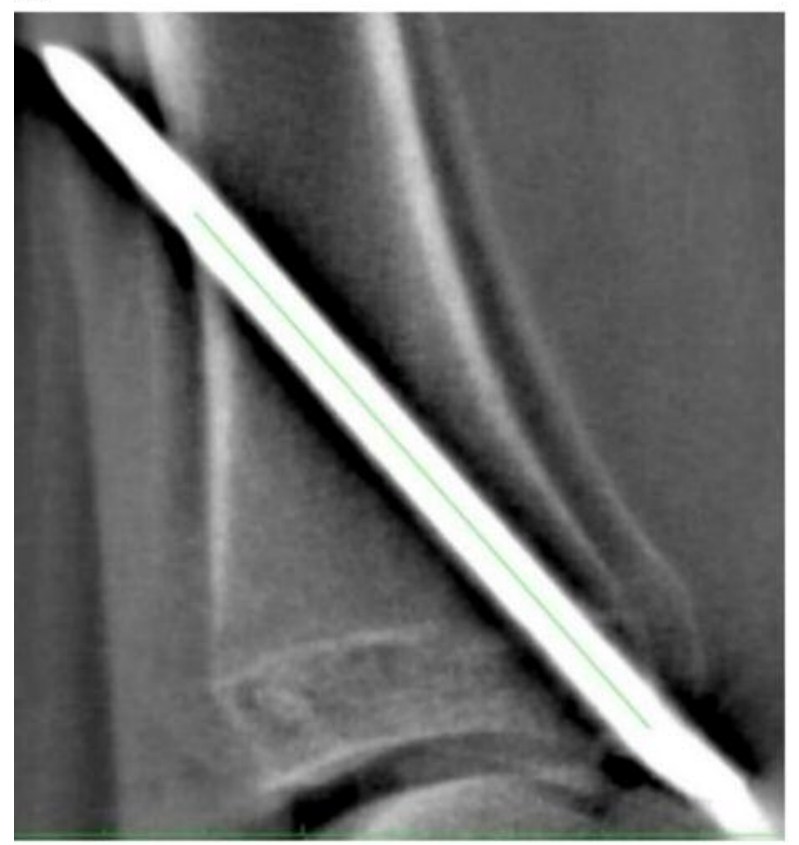

Figure 4. Intraosseous distance: Length of the wire within the calcaneus (green line within the wire); maximal distance from posterior calcaneus cortex to the wire (green line right rectangular to the wire) (a). Length of the wire within the tibia (b).

\section{Discussion}

Percutaneous extra-articular freehand calcaneotibial K-wire fixation requires an experienced hand to execute in such a way as to avoid iatrogenic injury to the articular surfaces of the ankle and subtalar joints. The novel targeting device, utilized in the current study, was constructed to ensure safe wire placement, regardless of the experience and the skill of the surgeon.

The aim of this study was to compare the accuracy of freehand technique and the target device for retrograde calcaneotibial fixation in an uninjured bone and an unstable
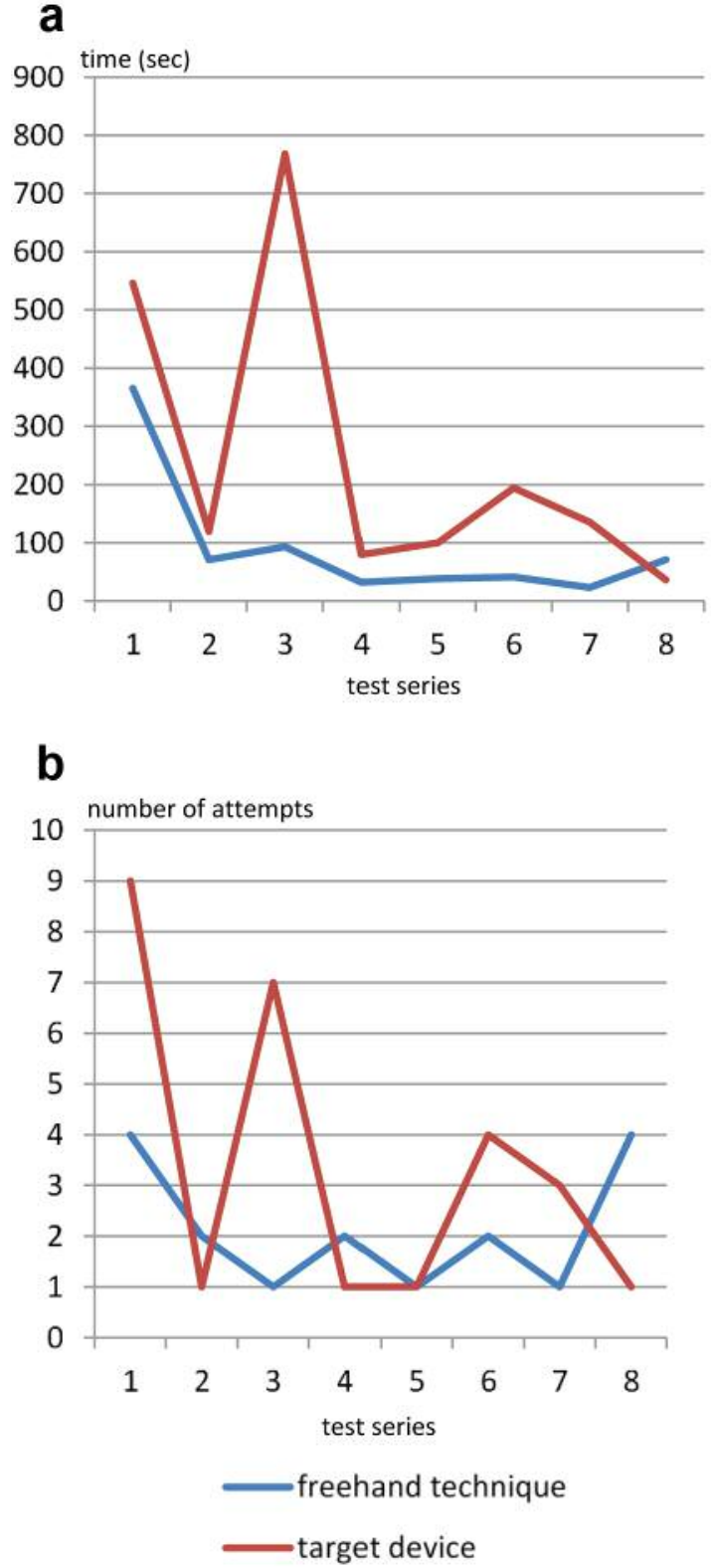

Figure 5. Learning curves for the time (sec) for drilling (a) and the number of drill attempts (b).

ankle fracture. The use of the targeting device requires significantly more time than the freehand technique. This can be explained by the experience of the performing trauma surgeon in freehand drilling.

The number of drilling attempts were not significantly different between freehand technique and the device $(p<0.794)$. It was shown that osseous fixation with the target device requires significantly more time than with the freehand technique $(p=0.04)$. 
Table I. Comparison of the freehand drilling technique and of a target device for retrograde calcaneotibial fixation.

\begin{tabular}{lcccccc}
\hline & $\begin{array}{c}\text { Intact } \\
\text { bone }\end{array}$ & $\begin{array}{c}\text { Bimalleolar } \\
\text { fracture }\end{array}$ & $\begin{array}{c}\text { Intact bone and } \\
\text { bimalleolar fracture }\end{array}$ & $\begin{array}{c}\text { Intact } \\
\text { bone }\end{array}$ & $\begin{array}{c}\text { Bimalleolar } \\
\text { fracture }\end{array}$ & $\begin{array}{c}\text { Intact bone and } \\
\text { bimalleolar fracture }\end{array}$ \\
\hline Device & Yes & Yes & Yes & No & No & No \\
Positioning time (sec) average & 162 & 144 & 153 & 0 & 0 & 0 \\
Wire placement (sec) average & 378 & 116 & 247 & 140 & 43 & 92 \\
Overall time (sec) average & 540.5 & 260 & 400 & 140 & 43 & 2.3 \\
Number of drills average & 4.5 & 2.3 & 3.4 & 2.3 & 2.8 & 9.9 \\
Total number image intensifier shots & 27 & 8 & 17.4 & 15 & 4.8 & 92 \\
\hline
\end{tabular}

Table II. Comparison of the freehand drilling technique and of a target device with regard to the correct positioning of the K-wire.

\begin{tabular}{|c|c|c|c|c|c|c|}
\hline & $\begin{array}{l}\text { Intact } \\
\text { bone }\end{array}$ & $\begin{array}{l}\text { Bimalleolar } \\
\text { fracture }\end{array}$ & $\begin{array}{l}\text { Intact bone and } \\
\text { bimalleolar } \\
\text { fracture }\end{array}$ & $\begin{array}{l}\text { Intact } \\
\text { bone }\end{array}$ & $\begin{array}{l}\text { Bimalleolar } \\
\text { fracture }\end{array}$ & $\begin{array}{c}\text { Intact bone } \\
\text { and bimalleolar } \\
\text { fracture }\end{array}$ \\
\hline Device & Yes & Yes & Yes & No & No & No \\
\hline Tibial wire length (mm, avarage) & 52.3 & 40 & 45.3 & 54.3 & 70.5 & 63.6 \\
\hline Calcaneal wire length (mm avarage) & 41.5 & 38.2 & 39.7 & 41 & 42 & 41.4 \\
\hline \multicolumn{7}{|l|}{ Sagittal plane (lateral view) } \\
\hline Distance wire/ posterior calcaneal border (mm, avarage) & 9.2 & 10.7 & 9.9 & 14.3 & 14 & 14.1 \\
\hline Wire position in corridor & $4 / 4$ & $4 / 4$ & $8 / 8$ & $4 / 4$ & $4 / 4$ & $8 / 8$ \\
\hline \multicolumn{7}{|l|}{ Coronar plane (ap-view) } \\
\hline Distance wire/lateral border tibial joint surface ( $\mathrm{mm}$ average) & 8.8 & 16 & 12.4 & 6.3 & 8 & 7.2 \\
\hline width of the distal tibial joint surface (mm, average) & 30.5 & 33 & 31.8 & 30.75 & 30.3 & 30.5 \\
\hline Ratio (average) & 0.29 & 0.49 & 0.39 & 0.34 & 0.26 & 0.3 \\
\hline Wire exit/ tibial joint surface (mm, average) & 37 & 26.8 & 31.9 & 42 & 37.7 & 39.8 \\
\hline Wire position in corridor & $4 / 4$ & $3 / 4$ & $7 / 8$ & $4 / 4$ & $4 / 4$ & $8 / 8$ \\
\hline
\end{tabular}

Despite the small number of cases, the distance of the $\mathrm{K}$-wire in the tibia was significantly shorter when the device was used $(p<0.015)$. The intra-calcaneal length was not significantly different between both methods $(p<0.303)$. The target accuracy of the experienced surgeon was shown to be superior to the target device used without any training. The development of the target device was based on previously determined angles calculated on weight-bearing radiographs of healthy feet (18). On weight-bearing radiographs, especially in hypermobile patients, who tend towards flatfoot deformity, the hindfoot axis tends towards a valgus deformity. The reduction of ankle fractures, by contrast, most commonly forces the hindfoot into a varus position. These different hindfoot positions resulted in a new definition of the desired trajectory, in the sagittal plane. As the sagittal angle of the target device is variable around the axis of the targeting-block, it requires no change in the targeting device. In the coronal view, the K-wire missed the defined corridor in the calcaneus once. The wire entry was too lateral in this case. Again, the use of load-bearing radiographs to determine the correct corridor for wire placement is limited due to the valgus heel position in a standing position. For radiographic imaging of the ankle joint on an AP view, the hindfoot is

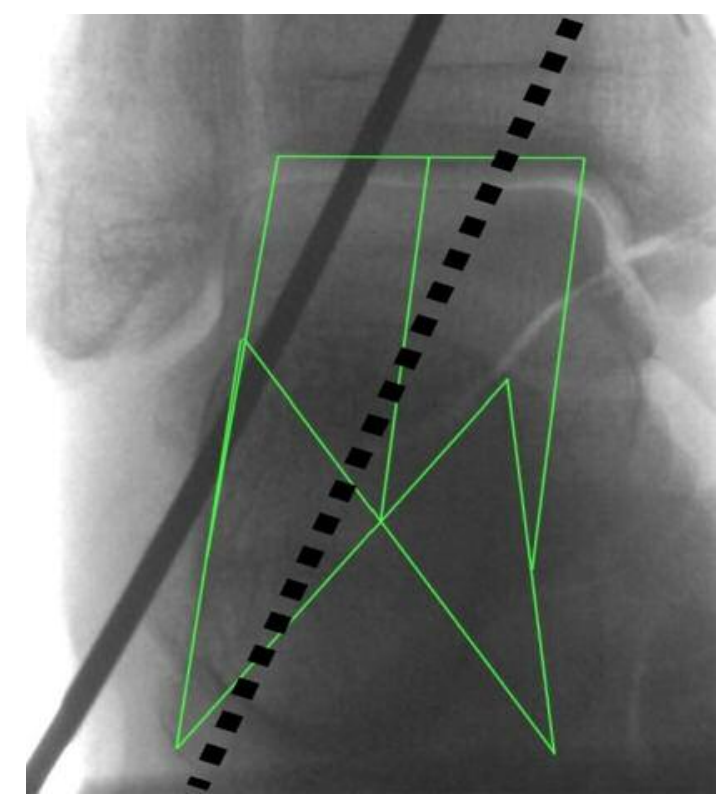

Figure 6. Intra-osteal K-wire position lateral to the calcaneal part of the corridor. A more medial entry of the wire would have led to a more central position in calcaneus (dotted line). Green lines: the mediolateral dimension of the corridor for the K-wire (18). 
held in $30^{\circ}$ of internal rotation. Thus, the coronal entry point has to be changed. A medial malposition has to be strictly avoided to protect the neurovascular bundle. Correct wire placement in the bony corridor is possible by choosing a more medial entry point with the aiming device (Figure 6). A modification of the device is not required, the calculated angles seemed to be correct. Instead more training for the operating surgeon is essential to ensure a correct positioning.

The results of the study are limited by the fact that the targeting device was used by the operating surgeon, for the first time. Therefore, the results were influenced by the inherent learning curve (Figure 5). This hypothesis is supported by shorter times and a decreased number of drill passes in the course of the entire test series. One can therefore expect shorter times with increasing use of the device. The small number of cases was another limitation of the study. As a result, many values in the small number of specimens tended to vary, but the results were not significantly different.

To the authors' knowledge, no other comparable biomechanical study on calcaneotibial fixation exists in the literature.

In summary, modification of the foot position in the targeting device is required in order to reliably avoid plantarflexion, and so prevent wire entry being excessively plantar. The entry angle of the wire, based on the data collected from native radiographs, proved experimentally to be correct. The device may be useful for correct placement of the K-wire for calcaneotibial fixation. Further research is necessary to determine if the targeting device simplifies the placement of the K-wire for an untrained surgeon after practicing with the device. Before clinical application however, the authors would advocate intensive training of the surgeon, in order to standardize the operative time, reduce the number of drill passes and optimize wire placement.

\section{Conflicts of Interest}

The Authors declare that there are no conflicts of interest regarding this work.

\section{References}

1 Lareau C, Daniels A, Vopat B and Kane P: Emergency department external fixation for provisional treatment of pilon an unstable ankle fractures. J Emerg Trauma Shock 8(1): 61-64, 2015.

2 Rammelt S, Heim D, Hofbauer LC, Grass R and Zwipp H: Problems and controversies in the treatment of ankle fractures. Unfallchirurg 114(10): 847-860, 2011.
3 Marvan J, Džupa V, Bartoška R, Kachlík D, Krbec M and Báča V: Kirschner Wire Transfixation of Unstable Ankle Fractures: Indication, Surgical Technique and Outcomes. Acta Chir Orthop Traumatol Cech 82(3): 216-221, 2015.

4 Carroll EA and Koman LA: External fixation and temporary stabilization of femoral and tibial trauma. J Surg Orthop Adv 20(1): 74-81, 2011.

5 Halanski $\mathrm{M}$ and Nooan KJ: Cast and splint immobilization: complications. J Am Acad Orthop Surg 16(1): 30-40, 2008.

6 Boyd AS, Benjamin HJ and Asplund C: Splints and casts: indications and methods. Am Fam Physician 80(5): 491-499, 2009.

7 Sanders D: Close Contact Casting vs. Surgery for Unstable Ankle Fractures. JAMA 316(14): 1451-1452, 2016.

8 Jaurequi JJ, Bor N, Thakral R, Standard SC, Paley D and Herzenberg JE: Life- and limb-threatening infections following the use of an external fixator. Bone Joint J 97-B(9): 1296-1300, 2015.

9 Haidukewych GJ: Temporary external fixation for the management of complex intra- and periarticular fractures of the lower extremity. J Orthop Trauma 16(9): 678-685, 2002.

10 Reff RB: The use of external fixation devices in the management of severe lower-extremity trauma and pelvic injuries in children. Clin Orthop Res (188): 21-33, 1984

11 Behrens F: General theory and principles of external fixation. Clin Orthop Res (241): 15-23, 1989.

12 Logan C, Ehrlichmann L, Ghorbanhoseini M and Kwon JY: Extra-articular calcaneo-tibial schanz pin stabilization for acute ankle trauma. The Orthopaedic Journal Harvard Medical School 18: 2080-2334, 2017.

13 Roukis TS: Extra-articular Ankle Stabilization: A Case Series. Foot Ankle Spec 3(3): 125-128, 2010.

14 Moss DP and Tejwani NC: Biomechanics of external fixation: A review of the literature. Bull NYU Hosp Jt Dis 65(4): 294-249, 2007.

15 Ferreira $\mathrm{N}$ and Marais LC: Prevention and management of external fixator pin track sepsis. Strategies Trauma Limb Reconstr 7(2): 67-72, 2012.

16 Bibbo $\mathrm{C}$ and Brueggeman J: Prevention and management of complications arising from external fixation pin sites. J Foot Ankle Surg 49(1): 87-92, 2010.

17 League AC, Parks BG, Oznur A and Schon LC: Transarticular versus Extraarticular Ankle Pin Fixation: A Biomechanical Study. Foot Ankle Int 29(1): 62-65, 2008.

18 Schröder M, Stüber V, Walendzik E, O’Loughlin PF, Krettek C and Gaulke R: Establishing an optimal trajectory for calcaneotibial K-wire fixation. Technol Health Care 23: 215-221, 2015. 